\title{
Risikoethische und entscheidungstheoretische Aspekte des Infektionsschutzes
}

\author{
Julian Nida-Rümelin $(\bowtie)$ \\ Staatsminister a. D., Ludwig-Maximilians-Universität, München, Deutschland \\ julian.nida-ruemelinalrz.uni-muenchen. de
}

Schlüsselwörter: Risikoethik · Deontologie $\cdot$ Entscheidungstheorie

\section{Die Realität des Risikos}

Objektivität des Risikos. In den 1990er-Jahren erschien ein dicker Band des größten Rückversicherungsunternehmens der Welt mit dem Titel „Risiko ist ein Konstrukt“ [1-3]. Der Titel entsprach zwar dem Zeitgeist, doch es war überraschend, ihn ausgerechnet von einem Versicherungsunternehmen präsentiert zu bekommen. Allerdings halte ich das für eine falsche Botschaft. Bei allem Respekt vor Ulrich Becks „Risikogesellschaft" [4-6] und vielen anderen Beiträgen zu dieser Debatte: Risiko ist eine Realität, nicht lediglich ein Konstrukt. Denn Schäden kann man beobachten, untersuchen und bewerten [7].

Dabei ist eine monetäre Bewertung, wie sie etwa Versicherungsunternehmen vornehmen, durchaus problematisch. Sie führt dazu, dass selbst bei großen Gefahren für Menschen zum Beispiel in der Region subsaharisches Afrika das Risiko als gering bewertet wird, während es in Tokyo, Osaka oder Südkalifornien als enorm hoch eingeschätzt wird. Warum? Weil in Japan und den USA die monetären Werte, die im Zweifelsfall etwa bei einem Erdbeben vernichtet würden, viel größer sind als in Afrika.

Dennoch ist diese Form von monetären Schäden objektiv bezifferbar. Etwas provokativ formuliert sind Wahrscheinlichkeiten ebenfalls einschätzbar und objektiv. Denn dafür gibt es sehr viele Datensammlungen, die über lange Beobachtungszeiträume erkennen lassen, wie häufig ein Schadensereignis auftritt. Daraus lassen sich sogenannte frequentistische Wahrscheinlichkeiten als Grenzwerte relativer Häufigkeiten bestimmen. Die resultierenden Angaben sind in vielen Fällen absolut verlässlich.

Wenn man sich auf die Einschätzungen der Menschen zu Risiken konzentriert, lässt sich sagen: Wichtig ist, dass die Einschätzungen in sich kohärent sind und zudem kompatibel mit den frequentistischen Daten. Wenn das nicht der Fall ist, steht man einer Form von Irrationalität gegenüber.

Subjektivität des Risikos. Ganz so wie bisher dargestellt ist die Situation allerdings nicht, denn es gibt ein subjektives Element in der Bestimmung des Risikos: die Bewertung. Umso stärker Bewertungen voneinander abweichen, umso komplexer wird die Situation. Und umso wichtiger sind kulturelle Verständigungsformen darüber, wie wir als Gesellschaft mit bestimmten Gefahren umgehen. 
Mit divergierenden Bewertungen muss man sich auseinandersetzen, denn die Gesellschaft muss handlungsfähig sein und mit bestimmten Gefahren umgehen. Diese Auseinandersetzung sollte in Form eines möglichst rationalen öffentlichen Diskurses erfolgen.

Im Hintergrund dieser Ausführungen steht eine Definition von Risiko als wahrscheinlicher Schaden, der beeinflussbar ist durch menschliches Handeln. Eine Anmerkung noch dazu: Die meisten Quantenphysiker sind der Auffassung, dass die Mikrowelt irreduzibel probabilistisch ist, wobei bis in die Gegenwart auch andere Interpretationen der Quantenphysik existieren. Jedenfalls ist umstritten, ob sich die probabilistische Struktur des Universums in der Mikrowelt in irgendeiner Form in der Makrowelt bemerkbar macht. Das heißt, man kann Probabilist sein bezüglich der Mikrowelt und Determinist bezüglich der Makrowelt.

Angenommen, die Welt wäre als Ganzes probabilistisch. Dann gäbe es in der Welt Wahrscheinlichkeiten. Wenn die Welt aber deterministisch ist, dann gibt es diese Wahrscheinlichkeiten in Wirklichkeit nicht. Dann stellt sich die Frage: Wie kann man behaupten, dass Wahrscheinlichkeiten etwas Objektives sind? Diese Frage soll hier nicht beantwortet werden. Sie soll nur darauf hinzuweisen, dass die Thematik komplex ist, wenn man sich tief gehend damit beschäftigt $[8,9]$.

\section{Ethik und Entscheidungstheorie des Risikos}

Risiko-Konsequentialismus. Auf den ersten Blick ist es absolut plausibel zu sagen: Wenn die Gesellschaft oder Unternehmen mit Risiken umgehen müssen, dann gilt es zu überlegen, mit welchen Wahrscheinlichkeiten treten welche Schäden wo, für wen, in welcher Region auf. Dann werden die Risiken aggregiert und das aggregierte Risiko im Sinne von wahrscheinlichem Schaden oder Erwartungswert des Schadens minimiert. Das wäre eine vernünftige und nachvollziehbare Risikopraxis. Diese Risikopraxis ist konsequentialistisch: Der moralische Wert einer Handlung wird anhand ihrer Konsequenzen beurteilt [10].

Kritik des Risiko-Konsequentialismus. Eine solche Risikopraxis kollidiert mit fundamentalen normativen Kriterien. Eines ist das Kriterium der Autonomie. Es gibt Risikoforscher, die sagen, es sei hochgradig irrational, dass Menschen freiwillige Risiken in weit höherem Maße akzeptieren als auferlegte Risiken [11]. Das geht bis zum Faktor Tausend. Wenn jemand sich beispielsweise entscheidet, Feuerwehrmann zu werden. Dann nimmt er freiwillig hohe Risiken auf sich. Wenn Menschen dagegen erfahren, ein Konzern bietet ein Produkt an, von dem ein geringes Risiko ausgeht, sagen sie: Das geht auf keinen Fall.

Üblicherweise wird ein solches Verhalten als ein Merkmal von verbreiteter Irrationalität interpretiert. Das ist falsch. Denn das Kriterium der Autonomie besagt: Letztlich entscheidet jeder selbst darüber, welche Risiken er für sich akzeptabel findet. Aus der Fremdperspektive ist das Verhalten nicht zu beurteilen [12].

Wenn im Recht der Suizidversuch straflos gestellt wird, dann heißt das: Man hat die Freiheit, sich selbst Risiken zuzumuten. Ein weiteres Beispiel: Man darf selbst entscheiden, ob man Kitesurfen macht und somit eine etwas erhöhte 
Ablebenswahrscheinlichkeit in Kauf nimmt, oder ob man auf Risikosportarten verzichtet. Individuelle Rechte zu haben, bedeutet immer auch, dass man entscheiden kann, ob man diese Rechte in Anspruch nimmt oder ob man beispielsweise einen Vertrag eingeht und auf sein jeweiliges Recht verzichtet. Das gehört zur Autonomie und ist keine Form der Irrationalität.

Ein weiterer Aspekt ist der der Gerechtigkeit. Angenommen, man könnte zehn Prozent der Bevölkerung, vielleicht gar der benachteiligten Bevölkerung, ein sehr hohes Risiko zumuten, um damit 90 \% der Bevölkerung von Risiken zu entlasten sodass das aggregierte Risiko insgesamt sinken würde. Die meisten Menschen würden sagen: Nein, das geht nicht, denn es wäre ungerecht.

Schließlich ein letzter Aspekt, der der Verhältnismäßigkeit, erläutert an einer interessanten Auseinandersetzung zwischen Wolfgang Schäuble, dem Bundestagspräsidenten, und Jürgen Habermas, dem bedeutendsten lebenden deutschsprachigen Philosophen. Dabei ging es um die allgemeine Abwägung zwischen Individualrechten. Steht Artikel 2 - ,Jeder hat das Recht auf Leben und körperliche Unversehrtheit" - über allen anderen Rechten abgesehen von Artikel 1 des Grundgesetzes - „Die Würde des Menschen ist unantastbar“? Habermas meint „Ja“, Schäuble meint „Nein“ [13]. In einer Interpretation hat insofern Habermas recht, weil es tatsächlich nicht geht, Menschen zu töten, um eine größere Anzahl an Menschen zu retten. Diese Auffassung hat auch das Bundesverfassungsgericht in seinem Urteil zum Luftsicherheitsgesetz vertreten. Eine solche Vorgehensweise wäre mit der Verfassungsordnung, vor allem mit der Unantastbarkeit der Würde, vielleicht auch mit Artikel 2, nicht verträglich. In einer zweiten Interpretation allerdings hat Schäuble recht: Bei einer generellen Abwägung steht der Gesundheitsschutz nicht über allem Anderen, weil wir ansonsten alle gesellschaftlichen Aktivitäten auf null fahren müssten. Die Gesellschaft muss aber handlungsfähig bleiben.

Risiko-Deontologie. Etwas vereinfacht dargestellt, ergibt sich aus einer umfassenden Abwägung: Man darf Risiken minimieren, also Optimierungskalkül betreiben, aber mit bestimmten deontologischen Einschränkungen, also unter der Voraussetzung, dass Würde, Leben, Rechte, Gerechtigkeit, gemeinschaftliche gesellschaftliche Praxis und gesellschaftlicher Zusammenhalt dadurch nicht gefährdet wird.

Diese Sichtweise allerdings führt zu einer Art Aporie, also einer Ausweglosigkeit, mit der sich die Philosophie seit Jahrhunderten beschäftigt. In der politischen Philosophie ist diese Aporie aufgelöst.

Dazu ein Beispiel, das sich auf die Risikopraxis übertragen lässt: Niemand ist von Natur irgendeiner anderen Person unterstellt. Wir sind alle gleich und frei. Das ist seit Thomas Hobbes das Dogma der Moderne. Andererseits können Regierungen uns zwingen und gar ins Gefängnis stecken. Wie legitimiert sich das? Die Antwort ist: Kontraktualistisch [6]. Das heißt, die These lautet: Vernünftige Menschen können unter bestimmten Bedingungen, etwa der Garantie von Individualrechten, einer Regierung zustimmen im allgemeinen Interesse, im Interesse jeder einzelnen Person. Das muss allerdings nicht nur postuliert, sondern irgendwie substanziiert werden. Im Falle der Risikopraxis kann sie nur substanziiert werden in der Weise, dass man sich öffentlich über Risiken und Akzeptabilität von Risiken austauscht und versucht - das wird nie ganz gelingen - einen möglichst weitgehenden gesellschaftlichen Konsens herzustellen. 
Deswegen bin ich skeptisch gegenüber einer Tendenz, die zu Beginn der Pandemie zu beobachten war: Spitzenpolitiker entzogen sich ihrer Verantwortung, indem sie auf die Virologen verwiesen: „Das sagt uns die Virologie“. Das aber ist der falsche Ansatz. Die Politik kann sich der Verantwortung nicht entziehen und die Virologie kann die Verantwortung nicht übernehmen. Denn die Infektionsschutzmaßnahmen sind nicht lediglich eine Sache der Experten, sondern müssen gesellschaftlich bewertet werden und im Idealfall im gesellschaftlichen Konsens getroffen werden. Es ist von allergrößter Bedeutung, dass die Zivilkultur intakt gehalten wird. Es muss Gewissheit herrschen, dass die Demokratie in Krisen trägt [14].

\section{Normative Aspekte des Infektionsschutzes}

Vor dem geschilderten ethisch-entscheidungstheoretischen Hintergrund soll nun eher stichwortartig auf konkrete Fragen des Infektionsschutzes eingegangen werden. Beispielsweise können Zulassungsverfahren für Impfstoffe oder auch für Medikamente nicht dadurch beschleunigt werden, dass man Menschen zur Teilnahme an Studien zwingt, auch wenn das für viele andere Menschen einen Vorteil bedeuten würde. Die Unantastbarkeit der Würde beinhaltet das Verbot, Menschen zu instrumentalisieren. Daher sind medizinische Studien und Zulassungsverfahren auf Freiwillige ausgerichtet. Wie schon dargelegt, ist Leben nicht verrechenbar.

Individuelle Rechte können durch Infektionsschutzmaßnahmen nur dann eingeschränkt werden, wenn diese Einschränkung nach klaren, nachvollziehbaren, wohlbegründeten Kriterien erfolgen, die durch Gesetze gestützt werden. In der öffentlichen Debatte werden häufig die Begriffe Kriterien und Maßnahmen nicht klar abgegrenzt und missverständlich genutzt. Entscheidend ist, dass die Kriterien eindeutig und zu rechtfertigen sind - und zudem mit anderen normativen Kriterien in der generellen Praxis übereinstimmen. Die Kriterien rechtfertigen Maßnahmen. Die resultierenden Maßnahmen können unterschiedlich sein, je nach Risikolage, je nach Region, je nach Gruppe, um die es geht.

In der öffentlichen Debatte wird - ein Übersprung aus der juristischen Debatte - viel von Verhältnismäßigkeit gesprochen. Häufig ist nicht völlig klar, warum die Verhältnismäßigkeit so wichtig ist. Verhältnismäßigkeit bedeutet nicht nur, dass man Risiken und die Risikoreduktion durch Maßnahmen vergleicht. Sondern man muss auch darauf achten, dass die jeweils getroffene Maßnahme mit der sonstigen Praxis vereinbar ist. Die normativen Kriterien müssen in sich stimmig sein. Betrachtet man generell die Risikopraxis, dann werden dort schwierige Fragen gelöst, indem Schwellenwerte definiert werden, bei denen das Risiko noch als akzeptabel gilt. Ein Beispiel liefert die Debatte um die Zulässigkeit der Kernenergie in den 1970er Jahren. In der Deutschen Risikostudie Kernkraft [15] hieß es, die Erhöhung der Ablebenswahrscheinlichkeit durch einen GAU (größtmöglicher anzunehmender Unfall) für Menschen, die in der Nähe eines Kernkraftwerks leben, läge in der Größenordnung von einem Millionstel. Das entspricht ungefähr der Wahrscheinlichkeit, vom Blitzschlag getroffen zu werden oder mit einem Flugzeug abzustürzen. Das Risiko galt deswegen als akzeptabel. Dabei soll hier gar nicht 
darüber diskutiert werden, ob die Berechnungen richtig waren. Es geht nur darum, dass Infektionsschutzmaßnahmen an anderen Umgangsweisen mit Risiko gemessen werden müssen. Wenn die Maßnahmen nicht in eine kohärente Risikopraxis passen, dann sind sie nicht zu rechtfertigen. Dann entstehen Probleme auch im öffentlichen Diskurs. Auf diese Probleme sollte man keinesfalls reagieren, indem man die Botschaft vermittelt: Für Diskussionen ist jetzt nicht der geeignete Zeitpunkt. Diese Botschaft gehört nicht in eine Demokratie, denn Demokratie ist dadurch charakterisiert, dass öffentliche Diskussionen immer zulässig sind und das politische Handeln begleiten und beurteilen.

Ich hoffe sehr, dass die demokratische Zivilkultur in dieser Pandemie gestärkt und nicht geschwächt wird. Da sind politische Akteure genauso wichtig wie die gesellschaftliche Verständigung, der gesellschaftliche Austausch. Es darf nicht passieren, was in einer anderen Krise passiert ist, nämlich der Migrationskrise: Am Ende ist die europäische Gesellschaft zerfallen in zwei ideologische Lager, die sich kaum mehr etwas zu sagen haben, sondern sich wechselseitig verachten. In einigen Ländern Europas hat dies dazu beigetragen die Demokratie erodieren zu lassen (Ungarn, Polen) oder sie zumindest zu gefährden (Italien, Frankreich, Großbritannien).

\section{Kooperation als Strategie}

Damit eine solche Krise wie die Covid-19-Pandemie kollektiv bewältigt werden kann, also nicht nur individuell jeder und jede für sich, bedarf es Bedingungen, die gesellschaftliche Kooperation sichern. Kooperation hat bestimmte Voraussetzungen. Kooperation heißt: Ich tue etwas, das für mich gar nicht optimal ist, das uns aber gemeinsam nützt, wenn wir uns allgemein in dieser Weise verhalten. Dazu ist intrinsische Motivation erforderlich [9, 16]. Diese intrinsische Motivation hat zumindest am Anfang der Krise eine massive Rolle gespielt. Anders ist etwa der Absturz des R-Faktors noch vor dem 22. März 2020 nicht zu erklären. Die Menschen hatten beschlossen, sich nicht nur selbst in Sicherheit zu bringen, sondern sich solidarisch zu zeigen, um beispielsweise die Großeltern zu schützen. Kooperation und Solidarität müssen in dieser Krise aufrechterhalten werden.

\section{Literatur}

1. Rückversicherung, B. (Hrsg.): Risiko ist ein Konstrukt. Knesebeck, München (1996)

2. Beck, U.: Risikogesellschaft. Auf dem Weg in eine andere Moderne, 22. Aufl. Suhrkamp, Frankfurt a. M. (2015)

3. Beck, U.: Weltrisikogesellschaft. Auf der Suche nach der verlorenen Sicherheit. Suhrkamp, Frankfurt a. M. (2006)

4. Julian Nida-Rümelin war mit dem Projekt „Strukturelle Gerechtigkeit“, Mitarbeiter Martin Rechenauer, an dem von Ulrich Beck geleiteten Sonderforschungsbereich „Reflexive Modernisierung“ (1999-2009) beteiligt, vgl. Nida-Rümelin J., Rechenauer, M.: Strukturelle Gerechtigkeit. Z. Politik, Neue Folge 56: 284-299 (2009) 
5. Nida-Rümelin, J., Weidenfeld, W.: Realität des Risikos. Zum vernünftigen Umgang mit Gefahren. Piper, München (2021)

6. Nida-Rümelin, J., Rath, B., Schulenburg, J.: Risikoethik. In: Grundthemen der Philosophie. De Gruyter (2012)

7. Nida-Rümelin, J.: Ethik des Risikos. Handbuch Angewandte Ethik, 2. Aufl., S. 806-830. Kröner, Stuttgart (2005)

8. Nida-Rümelin, J.: Unaufgeregter Realismus. Eine philosophische Streitschrift. Mentis, Paderborn (2018)

9. Nida-Rümelin, J.: Eine Theorie praktischer Vernunft. De Gruyter, Berlin (2020)

10. Nida-Rümelin, J.: Kritik des Konsequentialismus. Oldenbourg, München (1993)

11. Starr, C.: Social Utility versus Technological Risk. Science 165, 1232-1238 (1969)

12. Nida-Rümelin, J.: Wert des Lebens. In: Handbuch Angewandte Ethik, S. 832-861. Kröner, Stuttgart (2005)

13. Wolfgang Schäuble-Interview im Tagesspiegel 26. April 2020 und dazu kritisch: Habermas, J., Günther, K.: Kein Grundrecht gilt grenzenlos. Die Zeit Nr. 20/2020

14. Nida-Rümelin, J.: Die gefährdete Rationalität der Demokratie. Edition Körber, Hamburg (2020)

15. Die Deutsche Risikostudie Kernkraft wurde in den 1970er Jahren begonnen und immer wieder fortgeschrieben, Verlag TÜV Rheinland 1979, 1980, Phase B 1990 im Auftrag des Bundesministeriums für Forschung und Technologie, vgl. a. Die Beurteilung der Sicherheit deutscher Kernkraftwerke in der aktuellen fachwissenschaftlichen Debatte, Deutscher Bundestag WD 8-094-10 (2010)

16. Nida-Rümelin, J.: Structural Rationality and other Essays on Practical Reason. Springer International, Berlin (2019)

Open Access Dieses Kapitel wird unter der Creative Commons Namensnennung - Nicht kommerziell - Keine Bearbeitung 4.0 International Lizenz (http://creativecommons.org/ licenses/by-nc-nd/4.0/deed.de) veröffentlicht, welche die nicht-kommerzielle Nutzung, Vervielfältigung, Verbreitung und Wiedergabe in jeglichem Medium und Format erlaubt, sofern Sie den/die ursprünglichen Autor(en) und die Quelle ordnungsgemäß nennen, einen Link zur Creative Commons Lizenz beifügen und angeben, ob Änderungen vorgenommen wurden. Die Lizenz gibt Ihnen nicht das Recht, bearbeitete oder sonst wie umgestaltete Fassungen dieses Werkes zu verbreiten oder öffentlich wiederzugeben.

Die in diesem Kapitel enthaltenen Bilder und sonstiges Drittmaterial unterliegen ebenfalls der genannten Creative Commons Lizenz, sofern sich aus der Abbildungslegende nichts anderes ergibt. Sofern das betreffende Material nicht unter der genannten Creative Commons Lizenz steht und die betreffende Handlung nicht nach gesetzlichen Vorschriften erlaubt ist, ist auch für die oben aufgeführten nicht-kommerziellen Weiterverwendungen des Materials die Einwilligung des jeweiligen Rechteinhabers einzuholen. 